\title{
Limb infections in infancy presenting with pseudoparalysis
}

\author{
T. D. YUILLE \\ From the Paediatric Department, Royal United Hospital, Bath
}

\begin{abstract}
Yuille, T. D. (1975). Archives of Disease in Childhood, 50,953. Limb infections in infancy presenting with pseudoparalysis. Three cases of pseudoparalysis in infancy are reported in which the appearance of localized infection in the affected limb was delayed for a variable period after the onset of pseudoparalysis. Blood cultures on admission in all cases were positive, and bacteraemia due to the three organisms isolated is discussed.
\end{abstract}

Pseudoparalysis of a limb, with immobility, hypotonia, and hyporeflexia, is occasionally seen in infancy as a manifestation of a painful lesion in the limb. It must be distinguished from a true paralysis, indicating a neuropathy. In general a cause for the pseudoparalysis is apparent clinically or radiologically. Conditions which have been associated with this presentation are pyogenic arthritis and osteomyelitis, fractures and traumatic periostitis, subperiosteal haemorrhage of scurvy, and the osteitis of congenital syphilis. Soft tissue inflammation often occurs with osteomyelitis, but occasionally in infancy pseudoparalysis may be the sole sign of infection in bone.

Three cases of pseudoparalysis in infancy have been seen in a recent 6-month period at the Royal United Hospital, Bath, and at the time of admission, and for a variable period afterwards, no cause for the paralysis was apparent. Each had a blood culture on admission which showed bacteraemia. Subsequently a focus of infection did become apparent in the affected limb, but in no case was this in bone.

\section{Case reports}

Case 1. A 6-month-old infant with no previous illness presented with a 3-day history of poor feeding, vomiting, and irritability. One day before admission the right arm was noted not be be moving. On admission she was febrile and irritable. The right arm was immobile, hypotonic with depressed reflexes, and showed a full range of movement at all joints. There was no sign of an inflammatory lesion in the right arm, or elsewhere. Initial investigations showed a white cell

Received 1 April 1975. cell count $/ \mathrm{mm}^{3}$ of 17000 with a neutrophil leucocytosis, normal CSF, and normal $x$-ray of the right upper limb. A blood culture was taken. 24 hours later there was guarding and increased warmth of the right wrist, and treatment with flucloxacillin was started. The following day swelling and erythema developed over the palmar aspect of the right wrist, and the area was surgically explored. A subcutaneous abscess was found superficial to the muscle compartment, without deep communication and with no sign of bone or joint involvement. Haemophilus influenzae was subsequently cultured from the pus. On the fourth day, a pure growth of $H$. influenzae was obtained from blood culture taken on admission. The organisms were not typed. Ampicillin was given for 10 days, by which time the limb was entirely normal. Further $x$-ray showed no bone involvement. At follow-up several weeks later the baby was well.

Case 2. A 6-week-old infant was admitted with a history of oral moniliasis 6 days previously, followed 2 days later by irritability and poor feeding. One day before admission she stopped moving the right arm. At one week of age she had a severe purulent conjunctivitis treated for 2 weeks with local and parenteral gentamicin with complete recovery. On admission she was afebrile but irritable, particularly on movement at the right shoulder joint. Otherwise there was no sign of inflammatory lesion in the right upper limb, or elsewhere. The right arm was immobile and hypotonic with a full range of movement at all joints. Initial investigation showed a white cell count $/ \mathrm{mm}^{3}$ of 18500 with $46 \%$ neutrophils, normal CSF, and normal $x$-ray of the right upper limb. 12 hours after admission she became febrile, a blood culture was taken, and treatment started with ampicillin. Not until 24 hours after admission did guarding at the right shoulder joint develop, together with mild swelling and warmth overlying the shoulder joint. The swelling remained 
mild and appeared to be subcutaneous rather than a joint effusion. This settled after 3 days, as did the fever. The right arm gradually became more mobile and by 2 weeks was normal. On the sixth day a pure growth of pneumococcus was obtained from the blood culture taken soon after admission. The organism was sensitive to ampicillin which was given for 2 weeks. Further $x$-ray at 10 days showed no bone involvement. The baby was well at follow-up 2 months later.

Case 3. A 6-week-old infant, born by breech delivery, had been well until one week before admission when he developed fever and evidence of an upper respiratory infection. This was treated with tetracycline for 6 days. For 4 days before admission he showed reduced spontaneous movement of the left leg. On admission he was mildly febrile and mildly irritable, but not an ill-looking infant. Spontaneous movement and tone of the left lower limb were much reduced, particularly below the knee with foot drop. Range of passive movement at all joints was normal, and there was no sign of an inflammatory lesion in the affected limb. Initial investigations showed a white cell count/ $\mathrm{mm}^{3}$ of 9100 with a normal differential, normal CSF, and normal $x$-ray of the left lower limb. A blood culture was taken. Sciatic nerve palsy was considered, but there was no obvious cause for this and all virological tests subsequently proved negative.

Over the next 5 days there was very little change, with an intermittent mild fever and persistent poor tone and movement in the left lower limb. At times there was guarding of movement at the left hip, but this was not constant. On the sixth day after admission a warm swelling over the outer aspect of the mid-thigh on the left became apparent for the first time. This appeared to be a subcutaneous inflammatory swelling, well away from the sciatic nerve. Also at this time a pure growth of Bacteroides sp. was obtained from the admission blood culture. The significance of the organism in this clinical situation was uncertain, and a further blood culture was taken. A pure growth of Bacteroides sp. was again obtained after 5 days. By this time the swelling had diminished and the movement of the left leg was much improved.

The baby received no treatment after admission. The organism was sensitive to tetracycline with which he had been treated before admission. Follow-up several weeks later showed no abnormality. A pure growth of Bacteroides sp. on two occasions must be considered to indicate persisting bacteraemia, and it is reasonable to suppose that the swelling was the result of seeding of infection. Unfortunately, aspiration of the swelling was not attempted, and the organism was not further typed.

\section{Discussion}

These cases of pseudoparalysis of a limb were all associated with localized infection in the affected limb. Such infections usually affect bone or joint, but in these cases the infection appeared to be confined to the soft tissues. In Case 2 the signs of localized infection were minimal, and this may possibly have involved the shoulder joint. It is of interest that bacteraemia was present before clinical signs of localized infection became apparent, other than pseudoparalysis itself. It is thought unlikely that the signs which subsequently developed were missed initially, as the author examined the patients within a few hours of their admission. No other focus of infection was found on admission, though in each case there was a history of illness for a few days before admission. This suggests that bacteraemia preceded the onset of pseudoparalysis and that the localized infection which developed was due to seeding of infection from the blood. Pseudoparalysis was the first sign of this infection.

In each case the blood culture was taken with the usual aseptic precautions, and the organisms isolated are considered unlikely contaminants. $H$. influenzae and pneumococcus were isolated from Case 1 and Case 2, respectively. In a large series of febrile children in Boston, U.S.A., (McGowan et al., 1973) it was found that pneumococcus and $H$. influenzae B were organisms most commonly isolated from blood cultures. Localized limb infection associated with pneumococcal bacteraemia may take the form of cellulitis (Burke et al., 1971) or arthritis (Nelson and Koontz, 1966). Pneumococcal bacteraemia in the absence of a focus of infection is well known (Burke et al., 1971; Myers et al., 1974) and may have preceded the onset of pseudoparalysis in Case 2. $H$. influenzae as a cause of soft tissue infections in the extremities, as occurred in Case 1, appears to be increasing in frequency (Minnefor, Murray, and Davis, 1972), as is its occurrence in infections of bone and joint in young children.

Case 3 is of particular interest in view of the interval of several days between the onset of pseudoparalysis and the appearance of an inflammatory soft tissue swelling in the limb. That the swelling resulted from seeding of infection by Bacteroides sp. present in the blood must remain in doubt. However, it seems likely that this did occur, as bacteraemia was present at the onset of pseudoparalysis, the bacteraemia persisted, and improved mobility of the limb was associated with resolution of the soft tissue swelling. Subcutaneous infection with Bacteroides can occur after trauma, but there was no history of this in the case reported.

In a report of 4 cases and a review of 32 other cases of bacteroides infections in children (Sanders and Stevenson, 1968) pyogenic arthritis occurred 
several times, but cellulitis secondary to bacteroides bacteraemia appeared uncommon. The present case recovered completely without further antibiotic treatment after the organism was isolated. Recovery after inadequate treatment is not uncommon in infections arising in the genital tract of young women (Gelb and Seligman, 1970). However, infections in young children can be fatal, and usually are in newborn premature infants after intrauterine infection (Pearson and Anderson, 1967). It seems wise to give appropriate antibiotics to infants or children with bacteroides bacteraemia, particularly if persistent or in the presence of localized infection.

From these 3 cases it is suggested that bacteraemia preceded the onset of pseudoparalysis, and that this was the first manifestation of infection in the limb. They are unusual, as in at least 2 cases the infection was confined to the soft tissues and in none was bone involved. In the absence of a focus of infection on admission blood cultures were invaluable in isolating the organism.
I thank Dr. S. D. V. Weller for permission to report children under his care.

\section{REFERBNCES}

Burke, J. P., Klein, J. O., Gezon, H. M., and Finland, M. (1971). Pneumococcal bacteremia. American fournal of Diseases of Children, 121, 353.

Gelb, A. F., and Seligman, S. J. (1970). Bacteroidaceae bacteremia. fournal of the American Medical Association, 212, 1038.

McGowan, J. E., Bratton, L., Klein, J. O., and Finland, M. (1973). Bacteremia in febrile children seen in a 'walk-in' pediatric clinic. New England fournal of Medicine, 288, 1309.

Minnefor, A. B., Murray, J. J., and Davis, P. H. (1972). Hemophilus influenzae cellulitis of the lower extremity. American fournal of Diseases of Children, 124, 920.

Myers, M. G., Wright, P. F., Smith, A. L., and Smith, D. H. (1974). Complications of occult pneumococcal bacteremia in children. Fournal of Pediatrics, 84, 656.

Nelson, J. D., and Koontz, W. C. (1966). Septic arthritis in infants and children. Pediatrics, 38, 966.

Pearson, H. E., and Anderson, G. V. (1967). Perinatal deaths associated with bacteroides infections. Obstetrics and Gynecology, 30, 486.

Sanders, D. Y., and Stevenson, J. (1968). Bacteroides infections in children. Fournal of Pediatrics, 72, 673.

Correspondence to Dr. T. D. Yuille, The Grace Maternity Hospital, 5821 University Avenue, Halifax, Nova Scotia, Canada. 